\title{
El paradigma del deletreo: Avances en el desarrollo de interfaces cerebro-computadora aplicadas al deletreo de palabras
}

Graciela Ramírez-Alonso

Kathia Rascón-Cervantes

Alain Manzo-Martínez

Facultad de Ingeniería

Universidad Autónoma de Chihuahua

Email: galonso@uach.mx

\section{RESUMEN}

Se presenta una revisión de las diferentes estrategias metodológicas que se han implementado en los últimos años para el desarrollo de interfaces cerebro-computadora (BCI por siglas en inglés) centrada en el tópico de deletreo de palabras por medio del análisis de señales electroencefalográficas (EEG). La primera parte del trabajo hace referencia a las bases de datos que se han generado por diferentes laboratorios y universidades. La segunda parte explica la metodología que siguieron diferentes autores, así como los algoritmos de procesamiento de señales y los modelos de clasificación utilizados para el reconocimiento de caracteres. La literatura indica que se han alcanzado resultados en porcentaje de exactitud superiores al 90\%, lo que demuestra los grandes avances que se han logrado en esta área.

Palabras Clave: Paradigma del Deletreo, Señales EEG, Procesamiento de Señales, Modelos de Clasificación.

\section{Introducción}

En las últimas décadas, gracias a los avances en tecnología y los estudios en el área médica, se han buscado diferentes soluciones a los problemas que se presentan en pacientes con enfermedades neurológicas mediante el desarrollo de interfaces cerebrocomputadora (BCI). El objetivo de estas interfaces, es que pacientes que sufren alguna discapacidad puedan interactuar con su entorno y comunicarse de manera independiente (Ramadan y Vasilakos, 2017) (Wolpaw J. R., 2003). Específicamente, es de gran interés el desarrollo de interfaces de comunicación BCI enfocadas en el paradigma del deletreo de palabras, el cual se basa en el estudio de señales cerebrales recabadas mediante electroencefalogramas EEG. Este paradigma fue creado por Farwell y Donchin (1988), y consiste en presentarle al paciente una cuadrícula de 6x6 caracteres alfanuméricos en una pantalla en donde el objetivo es deletrear letra por letra una palabra. Primero se le pide al paciente que preste atención al carácter que desea deletrear. Luego, en la cuadrícula, se intensifican las 6 filas y 6 columnas de 
manera aleatoria durante $100 \mathrm{~ms}$ con un periodo de $75 \mathrm{~ms}$ entre intensificaciones, esperando con esto, que cuando se intensifique la columna $\mathrm{y}$ la fila que contienen al carácter deseado las señales eléctricas del cerebro generen potenciales P300. Estos potenciales son ondas con deflexiones positivas que aparecen alrededor de los $300 \mathrm{~ms}$ después del estímulo visual (Blankertz, et al., 2006). La figura 1 muestra este proceso.

Una vez que se tiene el registro de la señal que contiene los potenciales P300, se procede a implementar diferentes algoritmos de procesamiento de señales para su filtrado, extracción de características y clasificación automática. Con el fin de estandarizar el desempeño de los sistemas enfocados al desarrollo de interfaces de comunicación BCI, los investigadores han generado diferentes bases de datos, así como también, han implementado diferentes modelos y algoritmos para reconocer correctamente los caracteres a deletrear. En este artículo se describen las bases de datos más importantes que se han utilizado en los diferentes trabajos revisados, además de algunos de los modelos que han logrado buenos resultados en la etapa de clasificación de caracteres.

\section{Sistema de deletreo P300}

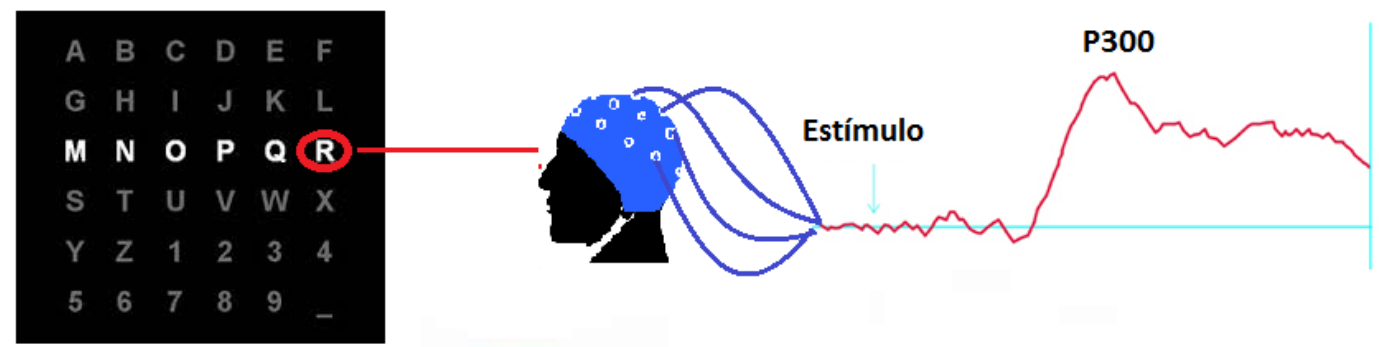

Figura 1. Ejemplo de cuadrícula 6x6 usada en un sistema de deletreo P300; 300 ms después de que se ilumina el carácter de interés, la lectura del EEG captará la deflexión positiva.

\section{Desarrollo}

\section{Base de datos de BCI Competition II y III}

Estas bases de datos fueron proporcionadas por el departamento de salud del laboratorio BCI del centro Wadsworth, New York en el año 2003 y 2005 respectivamente (Blankertz, et al., 2004) (Blankertz, et al., 2006). En ellas, se representa un registro completo de potenciales evocados P300 de dos sujetos, recabados con el software BCI2000. A los usuarios se les presentó una cuadrícula de 6x6 con 36 caracteres alfanuméricos como se muestra en la figura 1. Los usuarios debían de prestar atención a los caracteres de manera secuencial para formar una palabra previamente definida. Las seis filas y las seis columnas de la matriz se intensificaron aleatoriamente a un ritmo de $5.7 \mathrm{~Hz}$. Las señales fueron recabadas de 64 canales, filtradas con un pasa bandas de 0.1-60 Hz y digitalizadas a $240 \mathrm{~Hz}$. Estas bases de datos se encuentran disponibles http://www.bbci.de/competition/ii/ y http://www.bbci.de/competition/iii/\#data set ii. 
Base de datos del laboratorio de procesamiento de señales neuronales en Singapore

Al igual que la base de datos anterior, la base de datos de Singapore se generó a partir de presentarle al usuario una matriz de 6x6 caracteres alfanuméricos (Thulasidas, Guan, y Wu, 2006). El sistema con el que se adquirió esta base de datos se basó en el amplificador Neuroscan llamado SynAmps2, que tiene 64 canales monopolares y cuatro bipolares. Los canales bipolares son usados para monitorear el movimiento de ojos o parpadeo durante la adquisición de los datos. El software Neuroscan canaliza los datos en modo de servidor con un puerto TCP/IP y los recolecta con el mismo software en distintas máquinas a $250 \mathrm{~Hz}$. Durante la fase de recopilación de datos, a los sujetos se les presenta una secuencia de 41 caracteres en 10 rondas de 12 estímulos visuales en donde cada ronda hay un periodo de descanso de medio segundo.

Base de datos del laboratorio de la Universidad de Manitoba, Canadá

En este experimento se les presenta a los usuarios una pantalla de 7 regiones con grupos de 7 caracteres, tal como se muestra en la figura 2 (Fazel-Rezai y Abhari, 2009). Este nuevo paradigma trabaja con dos niveles, en el primero se le pide al usuario prestar atención al carácter que desea deletrear mientras se intensifican aleatoriamente cada uno de los 7 grupos. En el segundo nivel, los caracteres del grupo detectado son distribuidos en otras siete regiones. En este nivel, de manera similar al primero, las diferentes regiones son intensificadas mientras el sujeto se concentra en sólo una de ellas. Esta base de datos fue adquirida a partir de 10 sujetos, por cada sujeto se recabó la señal de 30 caracteres por medio de tres canales en donde las señales se digitalizaron a $500 \mathrm{~Hz}$.
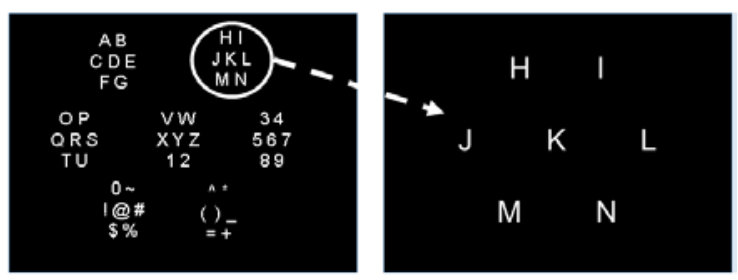

Figura 2. Paradigma propuesto por Fazel-Rezai y Abhari (2009).

Base de datos de la Universidad de Tecnología en Guangzhou, China

En este paradigma, se le presenta al sujeto una pantalla con una cuadrícula de 4x10 caracteres alfanuméricos como se muestra en la figura 3 (Chen y Zhang, 2017). Los caracteres se intensifican de manera aleatoria en donde uno de los estímulos corresponde al carácter deseado donde se espera como respuesta el potencial evocado P300. En este experimento, la duración de los estímulos o intensificaciones es de 100 ms, mientras que el periodo entre las mismas es de $30 \mathrm{~ms}$ y en cada intercambio de carácter a deletrear hay un periodo de 2.6 segundos. Las señales de 5 sujetos fueron recolectadas de 32 canales a $250 \mathrm{~Hz}$. 


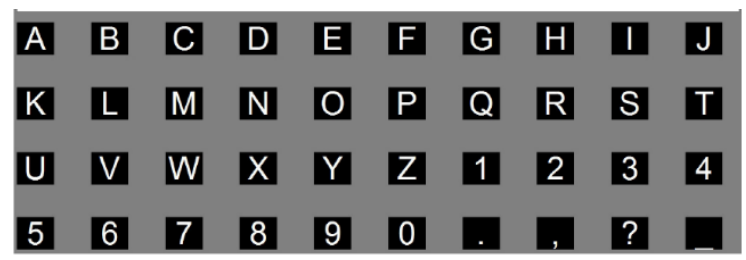

Figura 3. Interface utilizada por Chen y Zhang para el deletreo de palabras en base a señales P300.

Base de datos de la Universidad de Fargo, North Dacota

Para la adquisición de las señales se empleó el sistema inalámbrico EEG Motiv Research Edition, mientras que todos los códigos fueron escritos en MATLAB ${ }^{\circledR}$ (Obeidat, Campbell y Kong, 2015). La señal se adquirió de 14 canales con una frecuencia de muestreo de $128 \mathrm{~Hz}$, además, el equipo filtra la señal con un pasa bandas de $0.16-43 \mathrm{~Hz}$. Los datos adquiridos fueron de 17 sujetos en donde se le presenta al usuario una cuadrícula de 6x6 caracteres alfanuméricos. En esta investigación la modificación al paradigma anterior es como sigue, en lugar de intensificarse aleatoriamente las filas/columnas se intensifican los bordes izquierdo e inferior de la cuadrícula tal como se muestra en la figura 4. Cada estímulo dura un periodo de $120 \mathrm{~ms}$ con un intermedio de 120 ms entre intensificaciones.
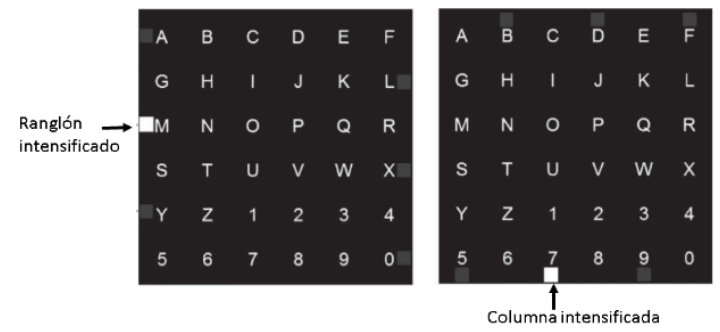

Fig. 4. Paradigma propuesto por Obeidat y Campell para el deletreo de palabras.

Base de datos de Ecole Polytechnique Fédérale de Lausanne en Lausanne, $\underline{\text { Switzerland }}$

En el experimento se utiliza la interfaz de Farwell y Donchin (ver figura 1) en donde se intensifican las filas y columnas de manera aleatoria por $100 \mathrm{~ms}$ y con un intervalo entre intensificaciones de $100 \mathrm{~ms}$ (Mirghasemi, Shamsollahi, y Fazel-Rezai, 2006). Los caracteres a deletrear por dos sujetos son elegidos previamente y éstos se muestran en la parte inferior de la pantalla. Para recabar esta base de datos se eligieron ocho palabras en el idioma francés teniendo 44 caracteres en total. Esta información se recaba con el equipo Biosem Active 2 que digitaliza la señal a $2048 \mathrm{~Hz}$ con 32 canales.

Las bases de datos descritas anteriormente han sido utilizadas en diferentes investigaciones. En esta sección del documento se describen en orden cronológico varias de las técnicas que han 
implementado diversos autores para tratar el problema del deletreo. Estas varían en los canales utilizados para adquirir la señal EEG, tipo de filtro para procesar la señal, tamaño de sección de señal que contiene la onda P300 y algoritmos de clasificación.

2004, Kaper. El objetivo de la investigación fue participar en la segunda competencia BCI por lo cual se hizo uso de la base de datos BCI Competition II (Kaper, 2004). De las señales proporcionadas en esta competencia se tomó la información de 10 canales (Fz, Cz, Pz, Oz, C3, C4, P3, P4, PO7 y PO8). Se etiquetó el conjunto de entrenamiento con los valores de 1 y -1 para indicar presencia/ausencia de P300. Luego, se creó un nuevo conjunto de entrenamiento, donde se tomaron secciones de $600 \mathrm{~ms}$ posteriores a cada intensificación. Estas secciones se definieron como entradas al algoritmo clasificador. Como preprocesamiento se empleó un filtro pasa bandas con frecuencias de corte de 0.5-30 $\mathrm{Hz}$ y se normalizaron las señales al intervalo $[-1,1]$. Se hizo uso de 5 validaciones cruzadas empleando una máquina de soporte vectorial (SVM) con los parámetros de $\mathrm{C}=20.007$ y $\sigma=27.359$. Esto fue para la clasificación binaria, es decir, si existe o no el potencial P300. Posteriormente, se buscaron las filas más probables llevando un control de la frecuencia de cada una en los resultados arrojados por el clasificador como presencia de P300. De manera análoga se determinaron las columnas. Finalmente, con las filas y columnas, se determinaron los caracteres deletreados. El autor determinó que el menor número de repeticiones necesarias para lograr una buena clasificación es de 3 repeticiones por carácter con lo que se logró una exactitud del $80 \%$.

2006, Mirghasemi et al. En esta publicación se hace uso de la base de datos de BCI
Competition II. El objetivo que se plantea es emplear diversos métodos de preprocesamiento y clasificadores para determinar la dependencia o independencia de éstos en la detección de potenciales P300 (Mirghasemi, Shamsollahi, y Fazel-Rezai, 2006). De la señal proporcionada por la base de datos se toma la señal de 3 canales $(\mathrm{Cz}$, $\mathrm{Pz}$ y Fz). Como pre-procesamiento de la señal se aplican tres métodos: filtro pasa bandas con frecuencias de corte de [0.5 30] $\mathrm{Hz}$, filtro de mediana y método Facet. Antes de realizar el procesamiento se normalizan todos los datos al intervalo $[-1,1]$. Como entrada al algoritmo se toma la señal correspondiente a 144 muestras (equivalente a 600 ms) después de un estímulo. Para disminuir la dimensionalidad de las entradas, se implementa un análisis de componentes principales (PCA), reduciendo de 144 a 21 muestras. Las etiquetas asignadas a estas características están dadas por el conjunto $\{-1,1\}$ que indican la ausencia o presencia de P300. Finalmente, se implementan cuatro algoritmos de clasificación con y sin aplicar PCA: análisis discriminante lineal (FLD), FLD con kernel Gausiano (KFD), y redes neuronales con dos (2RNA) y tres (3RNA) capas. En la red neuronal de dos capas se emplea una función de activación tangencial para la primera capa y una lineal en la segunda. En el caso de tres capas se usa la función tangencial en las primeras dos capas y una lineal en la tercera. En ambas redes se toma un aprendizaje de gradiente descendente. La mejor exactitud lograda fue de $96.77 \%$ empleando 3 combinaciones de preprocesamiento - clasificador: FLD con el filtro pasa bandas, 2LPNN con filtro de mediana y método Facet.

2006 Thulasidas et al. En esta investigación se hace uso de la base de datos de Singapore considerando la señal de 25 canales 
(Thulasidas, Guan , \& Wu, 2006). Como pre-procesamiento se implementa un filtro pasa bajas. Con el fin de reducir la dimensionalidad de los datos, se usó PCA para transformar de 25 (número de canales) a 20. En el algoritmo de clasificación se empleó una máquina de soporte vectorial (SVM) con kernel Gaussiano. Las entradas al clasificador fueron las concatenaciones de los vectores de $500 \mathrm{~ms}$ posteriores a un estímulo. Los autores realizaron varios experimentos y concluyeron que la exactitud no aumenta al emplear más de diez repeticiones de la señal. Se observó que con siete repeticiones la exactitud promedio es de $90 \%$ y con 10 repeticiones es de $95 \%$.

2007 Yang. El autor emplea la base de datos BCI Competition II considerando la totalidad de la información proporcionada de los 64 canales de la base de datos (Yang, 2007). Como pre-procesamiento se emplea un filtro pasa bandas con frecuencias de corte de 0.4 y $20 \mathrm{~Hz}$, escalando los datos al rango $[-1,1]$. Se tomaron secciones de señal con los valores de los $667 \mathrm{~ms}$ posteriores a cada estímulo, esto para los 64 canales. Se etiquetaron las muestras de entrenamiento con las etiquetadas +1 y -1 para indicar la presencia/ausencia de P300. Se buscaron los canales que presentaron las mayores amplitudes ordenándolos de manera decreciente tomando los más representativos. Para el algoritmo de clasificación se implementó una máquina de soporte vectorial con kernel Gaussiano variando los parámetros de C y sigma. Los autores reportan una exactitud del $100 \%$ en la clasificación de caracteres empleando únicamente 4 repeticiones, sin embargo, sólo se infieren 19 diferentes caracteres y no los 36 de la cuadrícula.
2008 Rakotomamonjy. El autor utiliza la base de datos de BCI Competition III. Con el fin de elegir la mejor selección de canales se prueban tres conjuntos de ellos: el primer conjunto contiene todos los canales de la base de datos (64 en total), el segundo conjunto está conformado por 8 canales (Fz, $\mathrm{Cz}, \mathrm{Pz}, \mathrm{C} 3, \mathrm{C} 4, \mathrm{P} 3, \mathrm{P} 4 \mathrm{y} \mathrm{Oz}$ ) y por último un conjunto de canales seleccionados con base en una eliminación recursiva (Rakotomamonjy, 2008). Antes de comenzar a trabajar con la implementación de algoritmos se analiza el comportamiento gráfico de las señales, de este análisis se concluye que los potenciales evocados aparecen en el intervalo de 300 a 350 ms después del estímulo (equivalente a 12 muestras). Se establece una división del problema en dos partes, la primera consiste en una clasificación binaria para presencia/ausencia de la onda P300, y la segunda parte consiste en la predicción de los caracteres. Como pre-procesamiento de toda la señal se aplica un filtro Chebyshev de orden 8 con frecuencias de corte de 0.1 y $10 \mathrm{~Hz}$. Por cada canal se extrajeron todas las muestras entre 0 y 667 ms posteriores a una intensificación. De acuerdo con el conocimiento sobre donde aparecen los potenciales evocados se tomaron 14 muestras correspondientes al post-estímulo de una intensificación de cada canal. El autor entrena 17 SVMs cada una con un conjunto diferente de caracteres. Los porcentajes de una correcta clasificación en promedio para los dos sujetos fue de $73.5 \%$ tomando sólo 5 repeticiones y 96.5\% con 15 repeticiones. Además, al hacer una comparativa entrenando los clasificadores con las señales del sujeto A y probándolos con las del sujeto $B$, se obtuvo un bajo porcentaje de correcta clasificación del 26\%.

2009 Fazel-Rezai y Abhari. Esta investigación aborda el tema sobre el error que generan los estímulos de filas y columnas adyacentes a la deseada en el 
experimento propuesto por Farwell y Donchin (Fazel-Rezai y Abhari, 2009). Se afirma que ésta es la principal fuente de error en la detección de potenciales evocados P300. Para demostrar esta afirmación se trabaja con las bases de datos BCI Competiton II y la base de datos de Manitoba, Canadá. De la primera base de datos se toma la información de 5 canales (Fz, Pz, Cz, C1 y C2), mientras que de la segunda se toma la de 3 canales $(\mathrm{Fz}, \mathrm{Cz}$ y $\mathrm{Pz})$. En la parte de extracción de características se obtienen los coeficientes del wavelet Mexican-hat y sus promedios se emplean como entradas al clasificador. Con la finalidad de comparar los dos paradigmas y determinar si el nuevo paradigma propuesto por el instituto de Manitoba realmente es mejor, se usa la señal de la palabra P3A. Los autores mencionan que se realizaron experimentos con una variedad de clasificadores, sin embargo, no se habla de cuáles fueron o cuál arrojó los resultados expuestos. El porcentaje de aciertos logrado al emplear la primera base de datos del paradigma de Farwell y Donchin es de $63.3 \%$, mientras que con el paradigma por regiones es de $81.7 \%$. Con base en los resultados arrojados, el nuevo paradigma basado en regiones representa una mejora importante en la exactitud en la identificación del carácter deseado.

2011 Cecotti. En esta investigación se trabaja con la base de datos BCI Competition III. Se toma la información de los 64 canales disponibles, como preprocesamiento se hace un sub-muestreo de toda la señal con la finalidad de reducir el tamaño de los datos a analizar a la mitad (120 Hz), finalmente, se filtra la señal con un pasa bandas con frecuencias de corte de 0.1 y $20 \mathrm{~Hz}$ y se normaliza la señal (Cecotti, 2011). El autor implementa primero un clasificador para la detección de la respuesta P300 y un segundo modelo realiza el reconocimiento del carácter. Para implementar el primer clasificador que debe detectar las respuestas P300 se utilizan redes neuronales convolucionales (CNN) de 5 capas mientras que para el reconocimiento del carácter se emplean modelos probabilísticos. El autor reporta un porcentaje de exactitud promedio del 94.5\%.

2015 Obeidat. En este trabajo se hace uso de base de datos de Fargo, North Dacota tomando la información de los 14 canales de la base de datos (Obeidat et.al 2015). Con el fin de eliminar el ruido a cada electrodo, se le restó el promedio de las series de tiempo de todos los sensores. Enseguida, se tomaron segmentos de datos del intervalo 0 a $600 \mathrm{~ms}$ posterior a los estímulos considerando 144 segmentos. Cada segmento es una estructura bi-dimensional que contiene la información de la señal EEG registrada de 14 electrodos con 77 intensificaciones. El clasificador empleado fue el de análisis discriminante lineal bayesiano (BLDA). Para entrenar el algoritmo se empleó de cada participante los datos de 29 caracteres. Los valores arrojados por BLDA para cada segmento correspondiente a cada fila o columna se promediaron en las 12 iteraciones. El autor realiza una evaluación en términos de calificaciones subjetivas sobre fatiga, comodidad y estado de alerta. Se realizó una comparativa entre los resultados generados con esta modificación basada en los bordes (EP) contra los resultados al emplear las intensificaciones comunes de fila/columna (RCP). La exactitud medida de la clasificación de EP es de 93.3\% mientras que con RCP se obtuvo un $81.7 \%$. El grado de fatiga se evaluó antes y después del experimento con una escala entre 0 y 9. Respecto a la fatiga, después de usar el deletreador, los participantes informaron estar menos fatigados con un 1.14 para EP que con 3.14 para RCP. Por otro lado, hablando de mayor comodidad, se obtuvo un 8.3 de EP y un 6.4 de RCP. Finalmente, al evaluar el sección de mayor alerta se arrojó 
un 8.5 de EP y un 6.1 de RCP. Estos resultados indican una mejora en la exactitud, en la velocidad de deletreo y en la experiencia del usuario que incluye una menor fatiga, mayor comodidad y mayor grado de alerta.

2017 Chen y Zhang. En esta investigación se busca explorar las redes neuronales convolucionales (CNN) empleando dos diferentes criterios de paro: static stopping criterion (SSC) y dynamic stopping criterion (DSC). Para esto hacen uso de la base de datos Ecole Polytechnique Fédérale. En el pre-procesamiento se realiza un submuestreo para reducir los datos en una sexta parte, luego se aplica un filtro pasa bandas de $0.5 \mathrm{~Hz}$ a $20 \mathrm{~Hz}$ y finalmente, se normaliza la señal (Chen y Zhang, 2017). La topología de la CNN está compuesta por 5 capas. Con el fin de resolver el desequilibrio de las muestras positivas y negativas en el deletreador P300 (un P300 frente a 39 muestras que no contienen P300 en este sistema) se tomaron todas las muestras con P300 y el mismo número de muestras sin P300 para cada época de entrenamiento. El algoritmo de clasificación arroja dos salidas que pueden ser 0 o 1 , las cuales indican ausencia y presencia de P300 respectivamente. En este trabajo se muestran los resultados de la detección de P300 y del reconocimiento de caracteres empleando SSC y DSC. En la detección de las P300 se reporta una tasa de reconocimiento promedio de $84.4 \%$. En la clasificación de los caracteres se logró una exactitud media de $96 \%$ para SSC y de $94.8 \%$ para DSC, aunque la exactitud de SSC es mayor que la de DSC, los autores de este trabajo afirman que con un umbral apropiado DSC puede mantener un buen rendimiento.

La tabla 1 muestra a manera de resumen las diferentes investigaciones que se describieron anteriormente, enfocadas a la implementación de modelos para el deletreo de palabras con base al análisis de señales EEG P300. Como se puede observar, la mayoría de los resultados que se reportan superan el $90 \%$ de exactitud, lo que demuestra los grandes avances que se han logrado en el desarrollo de interfaces cerebro-computada BCI enfocadas al deletreo de palabras.

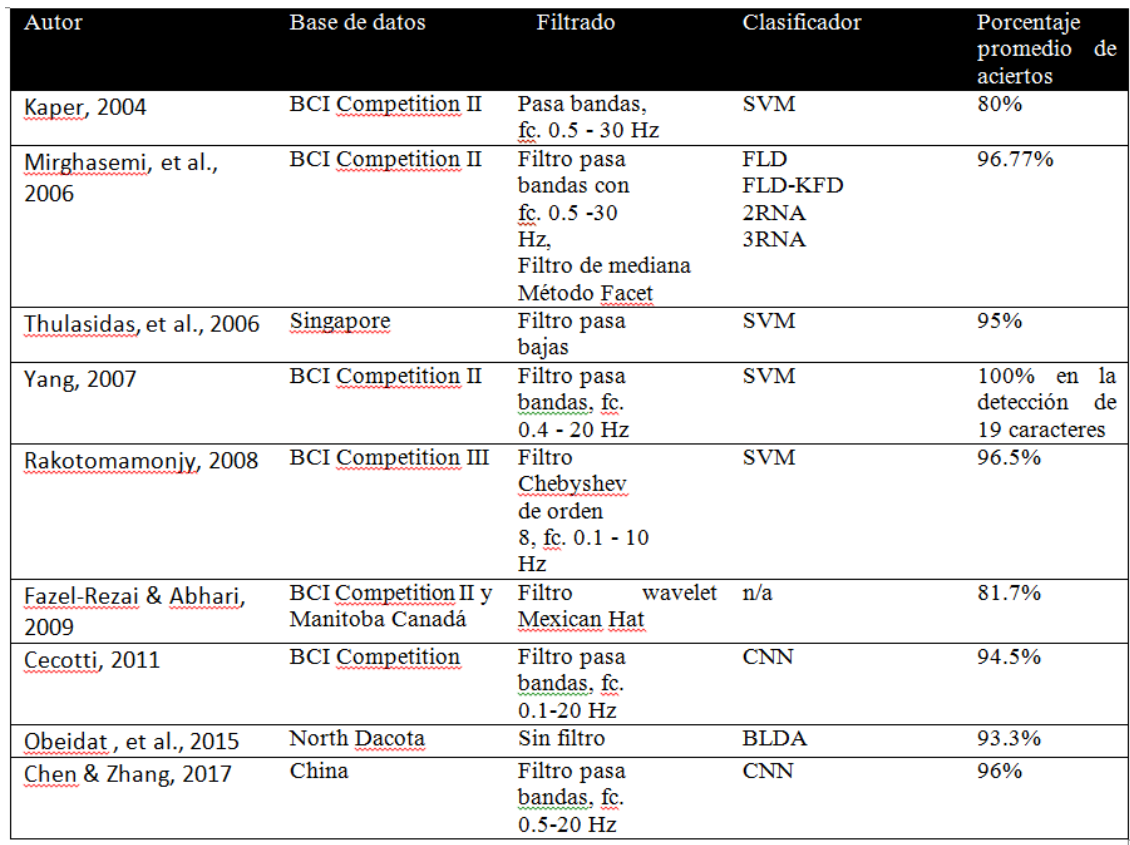

Tabla1. Resumen de artículos que han implementado diferentes algoritmos para el desarrollo de sistemas BCI enfocadas al deletreo de palabras 


\section{Conclusiones}

A la fecha, se han logrado muy importantes avances en el desarrollo de interfaces BCI. En este artículo se presentan aquellos modelos que han reportado los mejores resultados en la detección y clasificación de potenciales evocados P300 para el deletreo de palabras. De igual manera, se presentan las bases de datos que se han desarrollado para esta tarea. En la Facultad de Ingeniería de la Universidad Autónoma de Chihuahua, alumnos de las carreras de Ingeniería Matemática, Ingeniería en Sistemas Computacionales Hardware y el posgrado de
Maestría en Ingeniería en Computación se encuentran trabajando en diferentes algoritmos y modelos matemáticos para el procesamiento de señales EEG para el desarrollo de sistemas BCI. En estas aplicaciones se implementan diferentes algoritmos de procesamiento de señales y modelos de clasificación para la correcta interpretación de las señales EEG. Si se tiene interés en el desarrollo de aplicaciones en esta área pude contactarse con los autores de este artículo.

\section{Referencias}

Blankertz, B. y otros, 2006. The BCI competition III: validating alternative approaches to actual BCI problems. IEEE Transactions on Neural Systems and Rehabilitation Engineering, 14(2), pp. 153-159.

Blankertz, B. y otros, 2004. The BCI competition 2003: progress and perspectives in detection and discrimination of EEG single trials. IEEE Transactions on Biomedical Engineering, 51(6), pp. 1044-1051.

Cecotti, H. \&. G. A., 2011. Convolutional neural networks for P300 detection with application to braincomputer interfaces. IEEE transactions on pattern analysis and machine intelligence, 33(3), pp. 433-435.

Chen , Z. \& Zhang, X., 2017. Dynamic stopping in P300 speller with convolutional neural network. Shanghai, China, s.n., pp. 383-386.

Farwell , L. \& Donchin, E., 1988. Talking off the top of your head: toward a mental prosthesis utilizing event-related brain potentials. Electroencephalography and Clinical Neurophysiology, 70(6), pp. 510 - 523.

Fazel-Rezai , R. \& Abhari, K., 2009. A regionbased P300 speller for brain-computer interface. Canadian Journal of Electrical and Computer Engineering, 34(3), pp. 81-85.

Kaper, M. M. P. G. U. L. T. \&. R. H., 2004. BCI competition 2003-data set IIb: support vector machines for the P300 speller paradigm. IEEE Transactions on Biomedical Engineering, 51(6), pp. 1073-1076.
Mirghasemi, H., Shamsollahi, M. B. \& FazelRezai, . R., 2006. Assessment of Preprocessing on Classifiers Used in the P300 Speller Paradigm. New York, NY, USA, s.n., pp. 1319-1322.

Obeidat , Q. T., Campbell , T. A. \& Kong, J., 2015. Introducing the Edges Paradigm: A P300 BrainComputer Interface for Spelling Written Words. IEEE Transactions on Human-Machine Systems, 45(6), pp. 727738.

Rakotomamonjy, A., 2008. BCI competition III: dataset II-ensemble of SVMs for BCI P300. IEEE transactions on biomedical engineering, 55(3), pp. 11471154.

Ramadan , R. A. \& Vasilakos, A. V., 2017. Brain computer interface: control signals review. Neurocomputing, Volumen 223, pp. 26 - 44.

Thulasidas, M., Guan , C. \& Wu, . J., 2006. Robust classification of EEG signal for brain-computer interface. IEEE Transactions on Neural Systems and Rehabilitation Engineering, 14(1), pp. 24-29.

Wolpaw, J. R., 2003. Brain-computer interfaces: signals, methods, and goals. Capri Island, Italy,, s.n., pp. 584-585.

Yang, L. L. J. Y. Y. \&. L. G., 2007. An Algorithm to Detect P300 Potentials Based on F-Score Channel Selection and Support Vector Machines. In Natural Computation, Volumen 2, pp. 280-28

\section{Agradecimientos}

Los autores agradecen el apoyo de Prodep. Apoyo a la Incorporación de Nuevos PTC, convenio OF-17-8204. 